\title{
MACHINE LEARNING BASED ROAD DETECTION FROM HIGH RESOLUTION
} IMAGERY

\author{
${ }^{\mathrm{a}}$ Ye Lv, ${ }^{\mathrm{b}}$ Guofeng Wang, ${ }^{\mathrm{c}}$ Xiangyun $\mathrm{Hu}$ \\ ${ }^{a}$ School of Remote Sensing and Information Engineering, Wuhan University, P.R. China - 2010302590131@whu.edu.cn \\ ${ }^{\mathrm{b}}$ China Highway Engineering Consulting Corporation, Beijing, P.R.China - wgf@ checsc.com.cn \\ ${ }^{c}$ School of Remote Sensing and Information Engineering, Wuhan University, P.R. China - huxy@whu.edu.cn
}

\section{ICWG III/VII}

KEY WORDS: Road Extraction, Machine Learning, Image Segmentation, ROIs, Geometric Statistics Analysis, Road Feature

\begin{abstract}
:
At present, remote sensing technology is the best weapon to get information from the earth surface, and it is very useful in geoinformation updating and related applications. Extracting road from remote sensing images is one of the biggest demand of rapid city development, therefore, it becomes a hot issue. Roads in high-resolution images are more complex, patterns of roads vary a lot, which becomes obstacles for road extraction. In this paper, a machine learning based strategy is presented. The strategy overall uses the geometry features, radiation features, topology features and texture features. In high resolution remote sensing images, the images cover a great scale of landscape, thus, the speed of extracting roads is slow. So, roads' ROIs are firstly detected by using Houghline detection and buffering method to narrow down the detecting area. As roads in high resolution images are normally in ribbon shape, mean-shift and watershed segmentation methods are used to extract road segments. Then, Real Adaboost supervised machine learning algorithm is used to pick out segments that contain roads' pattern. At last, geometric shape analysis and morphology methods are used to prune and restore the whole roads' area and to detect the centerline of roads.
\end{abstract}

\section{INTRODUCTION}

As the increasing development of information industry and technology, the relationship between humans and geoinformation is tighter. Extracting geoinformation accurately and effectively has now been the shared goal of all information science researchers. Nowadays, photogrammetry, remote sensing and computer vision technologies can provide us with powerful tools to collect ,process and analyze massive amount of geo-spatial information, thus, giving a strong support for city development.

Extracting objects from remote sensing images has always been the main goal of computer vision. And because road has been the main subject in traffic analysis, city planning, disaster response and many other important issues, road extraction plays an important role in the research of city development. At present, high geometry resolution, high spectral resolution, and high radiation resolution remote sensing images give us more detailed information of our living environment, providing us with better quality research data, but in the mean time, it brings greater challenge as a result of greater computation complexity.

In recent twenty years, various kinds of road extraction methods have been proposed. Many research laboratories have done contributions to road extraction. Such as McKeown laboratory in America, "Amobe" Program in Switzerland,Karl-FranzensUniversitt Graz in Germany, Wuhan University, The PLA Information Engineering University and ATR laboratory of National University of Defense Technology in China and so on.

Roads' patterns are complex in high resolution images. Normally, roads' geometry feature, radiation feature, topology feature ,contextual feature are used for road extraction (Vosselman and Knecht, 1995). Indeed, there is a great number of roads detection methods, such as, mathematical morphology methods (Singh and Garg, 2013), template matching methods (Cheng et al., 2011), snake model methods (Yang and Zu, 2009), dynamic programming methods (Sujatha and Selvathi, 2015), segmentation methods (Song and Civco, 2004), edge detection (Sirmacek and Unsalan, 2010), perceptual organization methods (Gamba et al., 2006), machine learning methods (Wang et al., 2006), line detection methods, line tracking methods (Bicego et al., 2003) and so on. To improve the robustness of roads detection, various auxiliary data of different types is being used, for example, by using combination of Lidar cloud points and remote sensing images (Chen et al., 2009), or combination of DEM and remote sensing images (Hinz et al., 2001).

In this paper, the main detection strategy is shown as follows. Firstly, roads' ROIs are detected using canny edge detection and hough line detection method. Secondly, do image segmentation by mean-shift algorithm integrated with iterative marker-based watershed area merging algorithm. Thirdly, train a classifier by Adaboost algorithm using SIFT texture and color information, then use the classifier to select segments containing road patterns. Finally, by shape analysis and morphology methods, roads areas and center lines are extracted. Overall, image segmentation, feature extraction, machine learning, shape analysis and morphology methods are adopted for road detection in this paper. The main frame is shown in Figure 1.

\section{REGION OF INTEREST EXTRACTION}

\subsection{Introduction}

ROIs extraction contributes a lot to roads segmentation selection, this process helps to narrow down the searching space and cut down the time for feature calculation and analysis. In this part, Canny operator combined with Hough line detection algorithm is used.

Roads have contrast with their backgrounds, they are of low curvature, have small radiation variation and have topological connections with each other. As a result, gradient is very large at 


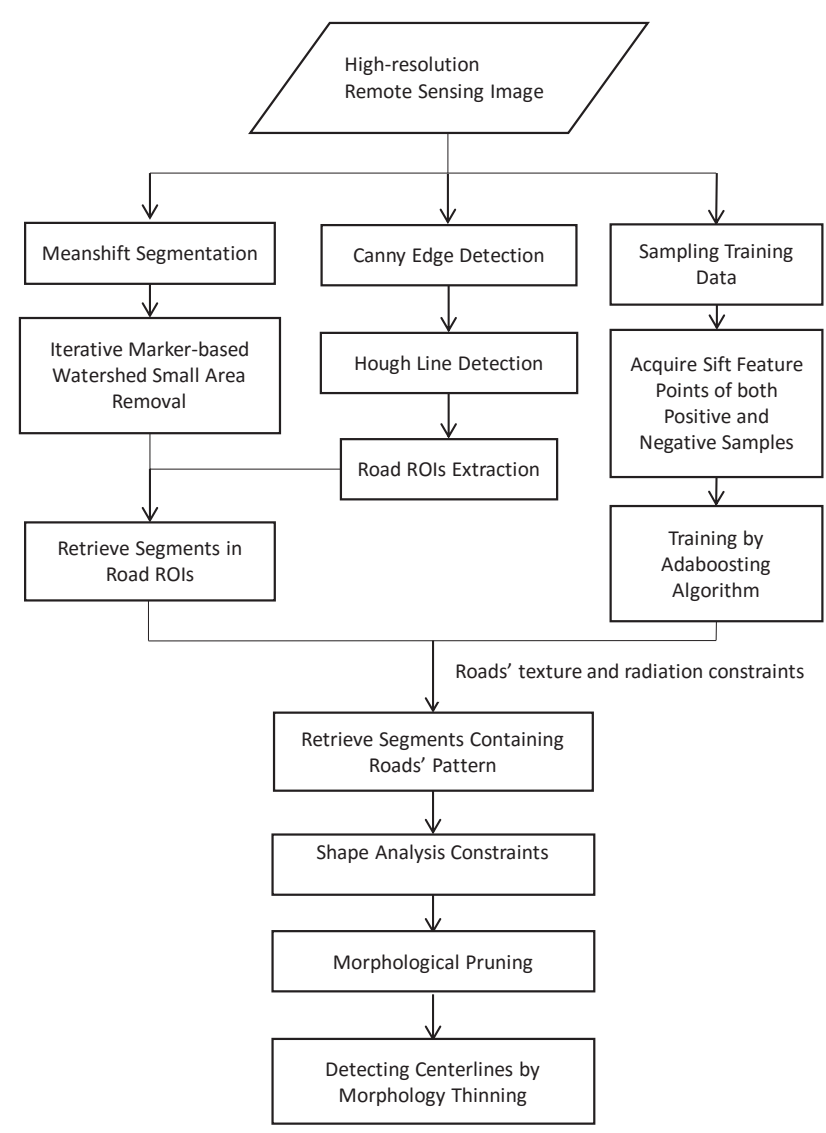

Figure 1. Road Extraction MainFrame

road edges, road lanes and isolation belts. In the meantime, these high gradient area has a length. By detecting these features, ROIs can extracted.

There are many edge detection operators, such as Roberts operator, Prewitt operator, Sobel operator, Kirsch operator, Nevitia operator, laplace operator, Canny operator, Marr-Hildreth operator and so on. Among them, Canny is regarded as the most robust operator.

Canny operator is brought out as a multi-level edge detector by John F.Canny in 1986 (J, 1986). This operator can depress noise in some degree, and effectively detect edges. So, it is of remarkable effect to detect roads edge features.

Hough transformation is an effective vote based parameter estimator. According to the points correspondence in image space and parameter space, Hough transformation changes the question of feature detection into accumulation statistics in parameter space. Hough transform can be adopted to detect arbitrary shapes (Ballard, 1987) such as, lines, circles, ellipses, parabolic curves and so on. By detecting lines, the strong indicators of roads existence.

\subsection{Experiment and Analysis}

Select 24 depth $2000 \times 900$ BMP image as research data. As shown in 2(a), the image contains many ground feature elements such as buildings, fields, roads, trees, grass, bare land and so on. The main roads width is about 28 pixels. In the image, roads edges, road lanes and cars can be seen.

To begin with, $3 \times 3$ Gaussian filter is applied to smooth the image. Then, the image of 3 channels is transformed into image

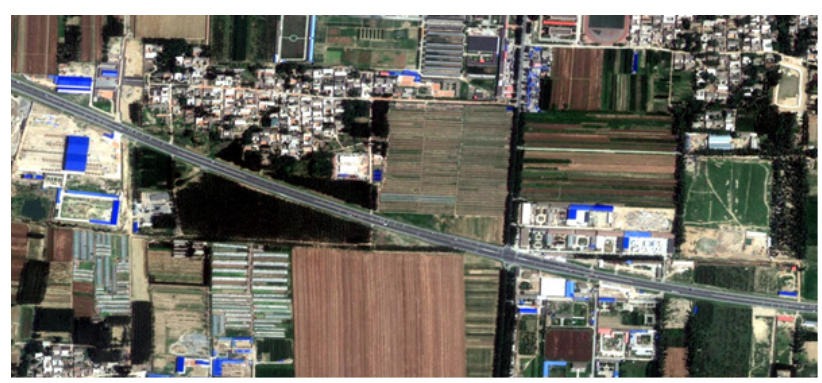

(a)

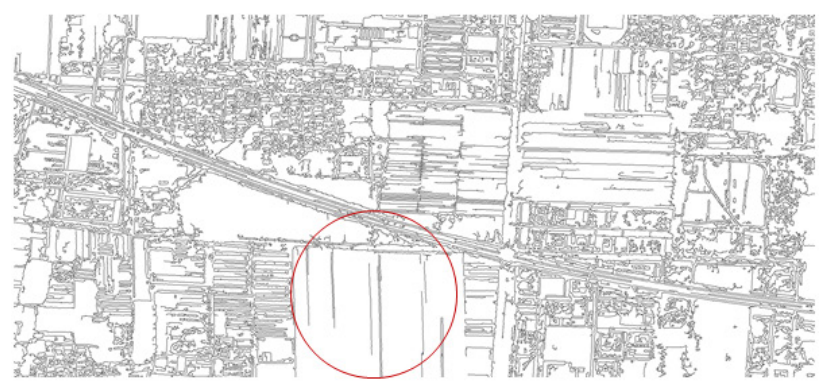

(b)

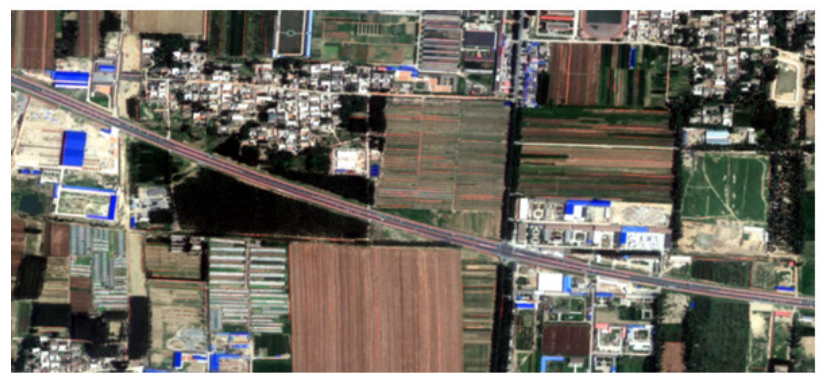

(c)

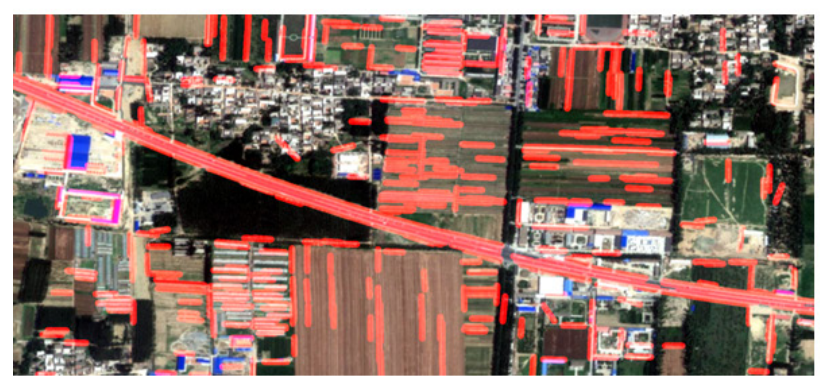

(d)

Figure 2. (a)Original image(b)Canny edge detection(c)Hough line segments detection(d)Hough line buffer

with 1 channel according to human visual property, the equation is shown in 1 below.

$$
\text { GreyValue }=0.299 R+0.587 G+0.114 B
$$

Then, adopt Canny operator, setting high threshold and low threshold parameter to be 125 and 40 separately. The edges are shown in Figure 2(b).

Designing a shifting window of $80 \times 80$ size, 40 shifting steps in both horizontal and vertical direction. Then, detecting lines in the window as window shifts in image. Requirements of line segments extraction from shifting window are as follows: 1) At most 3 pixels interval is allowed. 2) At least 25 pixels should be in a line segment. 3) In polar coordinate system, in which the 
hough lines are presented, step of length is 1 pixel, step of line direction is 0.5 degree. Result of Hough line detection result is shown in Figure 2(c).

It shows that major roads parts and lanes are detected. Besides, other line pattern in the fields and straight edges of buildings are detected. Based on these line segments, ROI buffers containing straight roads can be extracted, shown in Figure 2(d). The buffers cover most of the roads.

\section{IMAGE SEGMENTATION AND MERGING}

\subsection{Introduction}

Image segmentation partition the image into blobs of similar properties. It is an important process between image processing and image analysis. Image segmentation methods can be categorized into the following groups: 1 . Thresholding based method, such as image binarization, OSTU thresholding method. 2. Region based method, such as region growth method, splitting and merging method. 3. Edge based method, such as LoG operators, Canny operators. 4. Other specific theory based method, such as cluster analysis, fuzzy set theory, genetic coding method and so on. In this paper, Mean Shift segmentation algorithm is used for segmentation and Iterative Marker-based Watershed algorithm is used for small segments removal.

\subsection{MeanShift Image Segmentation}

Meanshift algorithm is based on non-parametric kernel density estimation, it is brought out first by Fukunaga (Fukunaga K, 1975 ) in 1975. Fukunaga named shift vector pointing to the maximum probility density to be meanshift vector. Comaniciu (D Comaniciu, 1999) proved the convergency of meanshift algorithm, and applied it to image smoothing, image segmentation (Comaniciu and Meer, 1997) and moving object tracking.

For color image, radius of grey values of three channels are correspond to color field parameter $r$, radius of pixel indexes of row and column are correspond to spatial field parameter $\mathrm{s}$. hs and hr are the bandwidth of $\mathrm{s}$ and $\mathrm{r}$ separately. The main steps are as follows. 1) Assign $y_{i, j}=x_{i}, \mathrm{i}$ is the index of pixels, $\mathrm{j}$ is the iteration times, beginning from $\mathrm{j}=1 ; 2)$ calculating $y_{i, j+1}=y_{i, j}+m_{h}(x)$ until $y_{i, j}$ converged to $y_{i, c}, \log$ value $y_{i, c}$. On above $m_{h}(x)$ is the meanshift vector; 3) Assign $z_{i}=y_{i, c}$; 4) In the range of hs and $\mathrm{hr}$, assign all the pixels that converged to $z_{i}$ to be $\left\{C_{p}\right\}_{p=1 \ldots m}$, that is, assign all the pixels converged to $z_{i}$ to be the same class. 5) Mark the class, and erase the segments that have too few pixels;

\subsection{Iterative Marker-Based WaterShed small Blobs Removal}

In the last 70s century, C. Digabel and H.Lantuejoul applied watershed algorithm in digital image processing. Due to the limitation of tremendous computation complexity, watershed algorithm was not widely used, but regarded as a binarization method. After the further research by Luc Vincent (Vincent and Soille, 1991) and S.Beucher (Beucher, 1994), computational complexity of watershed algorithm is optimized a lot and thus, watershed started to be widely used.

In 1992, Meyer introduced a non-parametric Marker-based Watershed algorithm (Meyer, 1992). The basic idea is to take gradient image as a landforms in geology. Each pixels value in the image represents the height above sea level. At each locally minimum height, prick a pole. Then, sink the model in the water. As the water is deeper and deeper, water begins to spread out from the poles forming local pools.

To eliminate over segmentation caused by small variation in gray value, gradient function can be modified as in equation 2 .

$$
g(x, y)=\max (\operatorname{grad}(f(x, y)), g)
$$

g stands for threshold, grad stands for calculating gradient operation. To eliminate noise and irregularity influence, an iterative non-parameter Marker-based Watershed algorithm is adopted to eliminate small blobs. Normally, it only takes two or three times iteration to achieve a good result. Iterative marker-based watershed algorithm is shown as follows. Firstly, set the threshold T for pixel number, such as 20 . Then, set iteration times to be $\mathrm{N}$, such as 2 or 3 . Thus, the threshold ti for each iteration time i can be defined, that is, $t i=T /(N-i+1)$, the input of iteration $i+1$ is the output of iteration i. As for pixels at the boundary of differen$t$ segments. They can be assigned to the nearby segment whose average pixel value is closest to them. The advantage of applying watershed merging strategy is that: 1) Merging starts from the pixel with minimum value contrast against it, it avoids looking for pixels that have closest value. 2) No need to look for adjacent segments and no need to manage the adjacency list. 3) Effective when dealing with lanes or cars on road. The disadvantage of this method is that objects with very small width will be lost. However, the roads width is large enough not to be erased.

\subsection{Experiment and Analysis}

Apply the meanshift filter on the color image, setting the hs to be 8 and hr to be 10 . In each segment, assign the average color of pixels in it to each pixel within. After applying the filter, the image will be a little blurred shown in Figure 3(a).

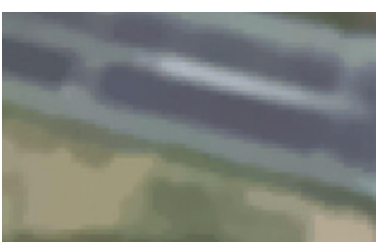

(a)

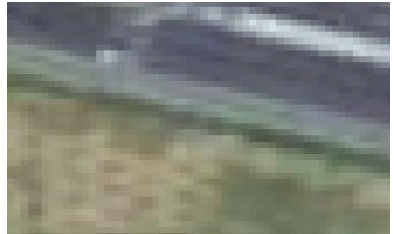

(b)
Figure 3. (a)Local meanshift filter result (b)Local origin image

Overall result is shown in Figure 4(a).

Merge the small color contrast segments according to equation 3 . $\operatorname{Src}(\mathrm{x}, \mathrm{y})$ is the image. $\mathrm{x}, \mathrm{y}$ is the coordinates of the point to be modified, $x^{\prime} y^{\prime}$ are the 4 neighborhood of pixel $(\mathrm{x}, \mathrm{y})$. loDiff and upDiff are two threshold. In the experiment, set both loDiff and upDiff to be 5 .

$$
\operatorname{src}\left(x^{\prime}, y^{\prime}\right)-\operatorname{loDiff} \leq \operatorname{src}(x, y) \leq \operatorname{src}\left(x^{\prime}, y^{\prime}\right)+u p D i f f
$$

The Figure 4(b) shown is the classification result. In the figure, different segments are shown in different color. There are many broken segments most of them are not to 5 pixels area. Owing to the lanes and cars, the road is hard to be classified into one single class, especially caused by the influence of white lanes, which segment the roads into irregular shapes. In addition, there 


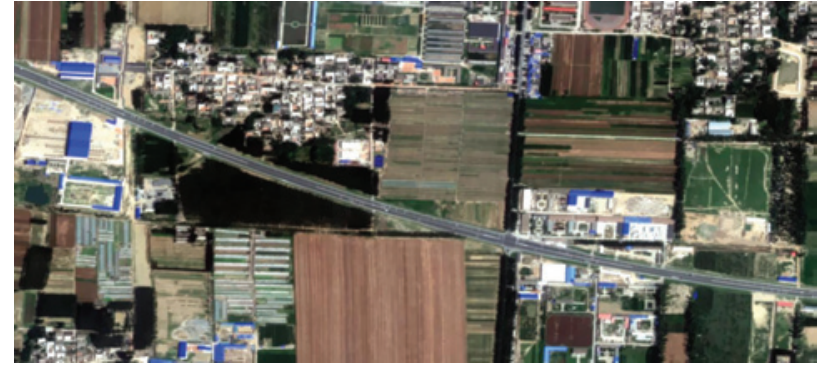

(a)

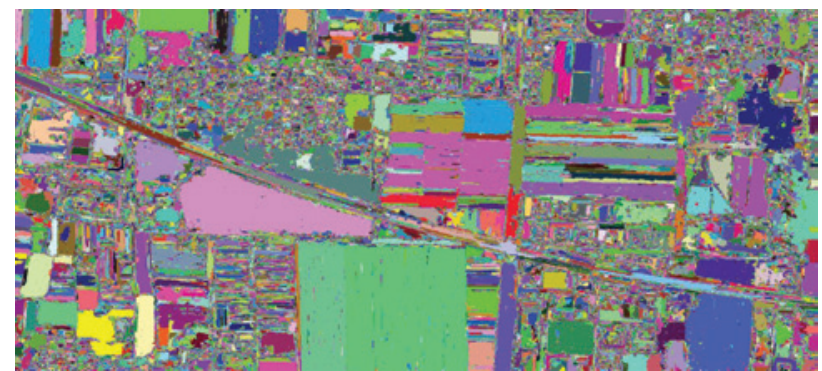

(b)

Figure 4. (a)Meanshift filter result (b)Meanshift image segmentation

is a gradual change in the radiation along the road, so, the road is partitioned into different parts.

Using method based on Iterative Marker-based Watershed, smal1 segments can be effectively eliminated, including unnecessary details such as cars. Setting threshold $\mathrm{T}$ to be 80 and iteration times $\mathrm{N}$ to be 2 . Some too narrow objects will be eliminated shown in Figure 5(a) and Figure 5(b).

Figure 5(c)(d) shows two times small segments removal effects. The color of the segment is the average color of the pixels within. The figure shows, segments whose pixel number is above 40 less than 80 will not be merged to other segments.

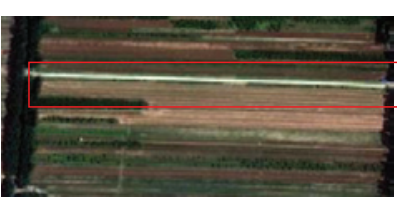

(a)

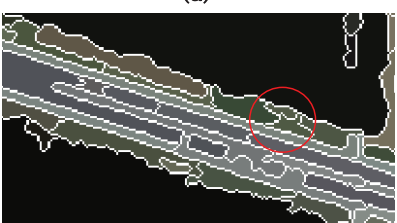

(c)

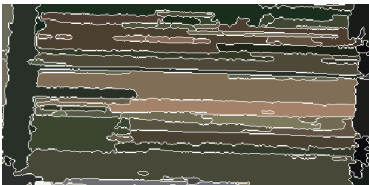

(b)

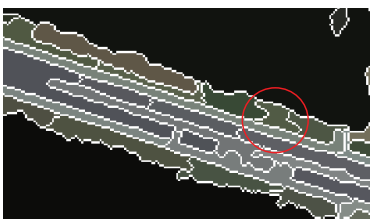

(d)
Figure 5. (a)Origin Image (b)First merging result (c)First merging result (d)Second merging result

Overall segmentation results are shown in Figure 6

After segmentation, extract the segments who has $60 \%$ area inside the ROI buffers. The result is shown in Figure 7, segments with line patterns are reserved as well as some other small segments which will be eliminated afterward.

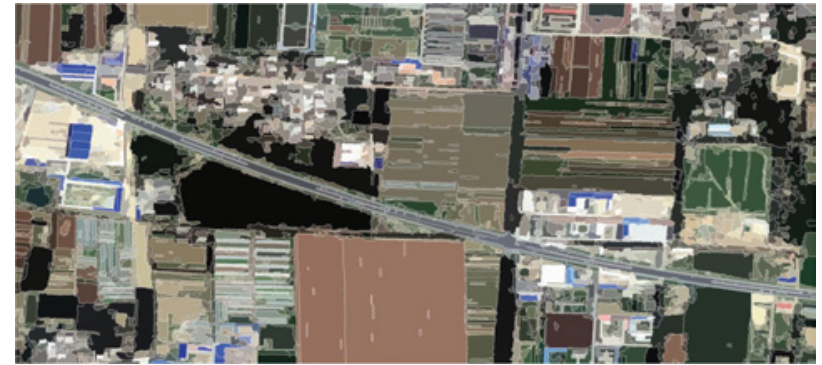

(a)

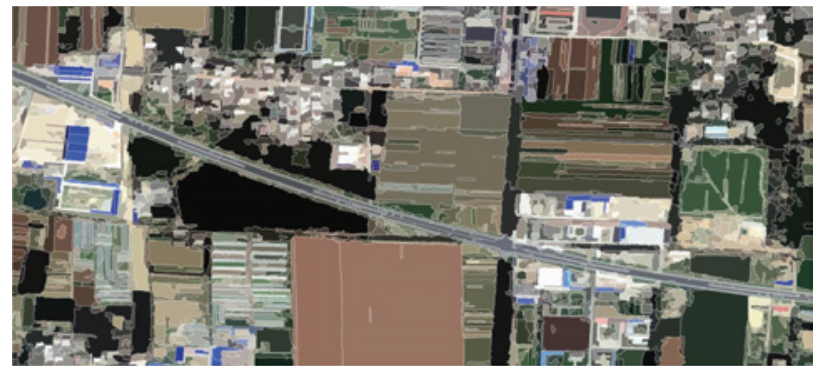

(b)

Figure 6. (a)First watershed iteration result (b)Second watershed iteration result

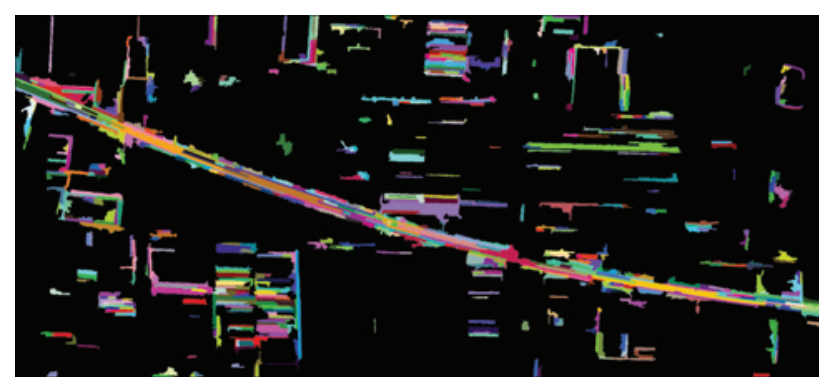

Figure 7. Segments in ROIs

\section{ROAD SEGMENTS EXTRACTION}

\subsection{Introduction}

Roads have unique texture patterns involving cars, lanes and passengers. To retrieve segments with road patterns, method that combines SIFT and AdaBoost algorithm is used. SIFT algorithm creatively computes key points according to DOG and constructs 128 dimensions local invariant feature descriptor to analyze the local feature pattern. With SIFT feature descriptor and 3 color channels, patterns of roads are sent to AdaBoost algorithm. After training the classifier, the classifier can be used to select road segments.

\subsection{Texture Analysis}

Texture analysis is one of the hottest issue, and is applied in many fields. Such as object detection, remote sensing image analysis, products checking and medical image analysis. Common extraction methods includes: construction method, statistics method, frequency spectrum method and model method.

SIFT is useful and effective in object detection and matching. It is scale and direction invariant, more scale invariant and multiscale spatial theories are illustrated in (Lowe, 2004) (Lindeberg, 
1994). Besides, it is adaptive to light variation and image distortion, and has high differentiating capability. SIFT has mainly two parts, they are key points detection and feature description.

As for key points detection. First, subsample the image using different scale Gaussian kernel function to get Gaussian image pyramid. Second, compute DOG by adjacent level images. Third, compare the points with neighbor points in current and adjacent level images, and set local extremes to be the key points and log their positions and scales. For better precision, curved surface fitting can be used.

As for descriptor representation, take the key point of its scale as center, then take $16 \times 16$ neighbor pixels and divide these 256 pixels into $4 \times 4$ sub-blocks. Calculate gradient direction histogram ( 8 main direction from 0 to 360 degree) for each sub-block. Then, rearrange the order forming $4 \times 4 \times 8=128$ dimensions vector.

\subsection{Adaboost Training}

In the field of machine learning, after Valiant published paper (Valiant, 1984). Many researchers turned focus on the PAC (Probably Approximately Correct) learning model.

Kearns and Valiant (Kearns et al., 1994) said that, in PAC learning model, if there exists a polynomial learning algorithm for concept recognition with high correct rate, then these concepts are strongly learnable. On the contrary, if the correct rate is only a little better than random guess, then these concepts are weakly learnable. Kearns and Valiant proved the equality of weakly learnable algorithm and strongly learnable algorithm, that is, weakly learnable model can be strengthened into strongly learnable algorithm.

In 1995, Freund and Schapire brought out Adaboost algorith$\mathrm{m}$. AdaBoost algorithm is a supervised learning algorithm, it cascades many weak classifiers into a very effective classifier. Though these weak classifiers may be simple, but only if their result is a little better than random guess, they can be cascaded into strong classifier with Boosting methods. Boosting algorithm has many variants, including Discrete AdaBoost, Real AdaBoost, LogitBoost and Gentle AdaBoost. To reduce the computation time, samples already being classified with very high confidence can be excluded from training set (Freund, 1995).

\subsection{Experiment and Analysis}

There are mainly four steps for classification. 1) Sample points selection.2) Feature descriptor construction.3) Classifier Training.4) Classification of the key points in ROIs.

1)Manually select positive and negative samples range from which samples are extracted. To improve the classifier, the sample types should be rich enough. A $2000 \times 2000$ image is used. In the range, SIFT key points extraction algorithm is applied to extract key points. The Figure 8(a)(b)(c) below shows the original sampling image, positive and negative sampling range.

2)Calculate the feature descriptor of the extracted key points in both positive and negative range. Then, add the value of three color channels to the end of the descriptor (Figure 9). Use this descriptor to extract positive and negative samples from positive range and negative range. Figure 9(d) shows SIFT key points, radius of the circle represents the scale and the radius direction represents the direction. The experiment is done by using OpenCV. SIFT parameters are set as follows. The number of layers in each octave is 3 , the contrast threshold used to filter out weak features is 0.01 , the threshold used to filter out edge-like features is 20 .

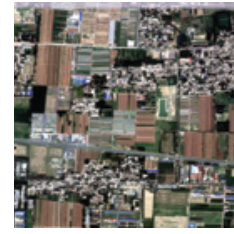

(a)

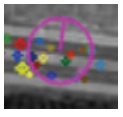

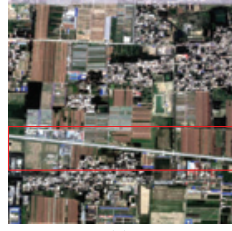

(b)

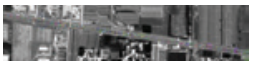

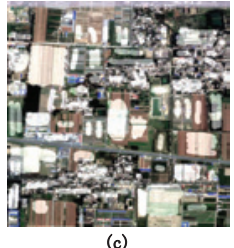

(c)

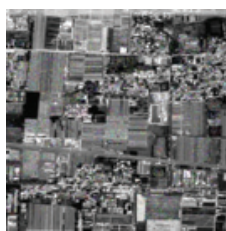

(f)
Figure 8. (a)Original image used for training classifier (b)Positive sampling range (c)Negative sampling range (d)Key point representation (e)Positive SIFT key point samples (f)Negative SIFT key point sample

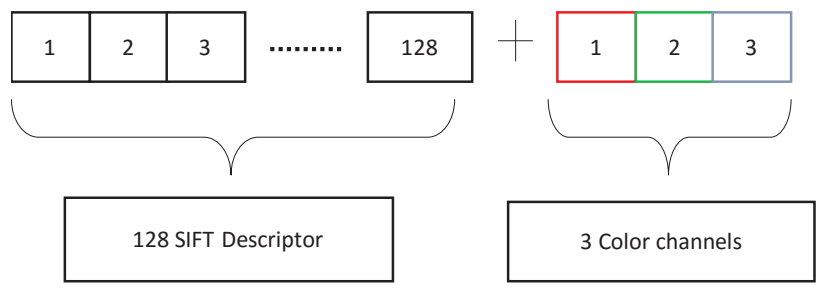

Figure 9. Descriptor

Sigma of Gaussian filter is 1.2. The second and the third parameter should not be too high in order to get enough training samples. Figure 9(e)(f) show the positive and negative sample key points separately. There are 1198 positive samples and 37748 negative samples.

3)Real AdaBoost is adopted, set the weak classifier to be 200, when any samples correct rate is above 0.999 , it is removed from the training set. After the classifier is retrieved, it can be applied to our original image. First, key points in road ROIs are detected, then the key points with road texture can be extracted using classifier. The result is shown in Figure 10. The green points are the key points classified as points with road texture pattern. Precision rate is very high, only 9 points are wrongly classified as non-road pattern points.

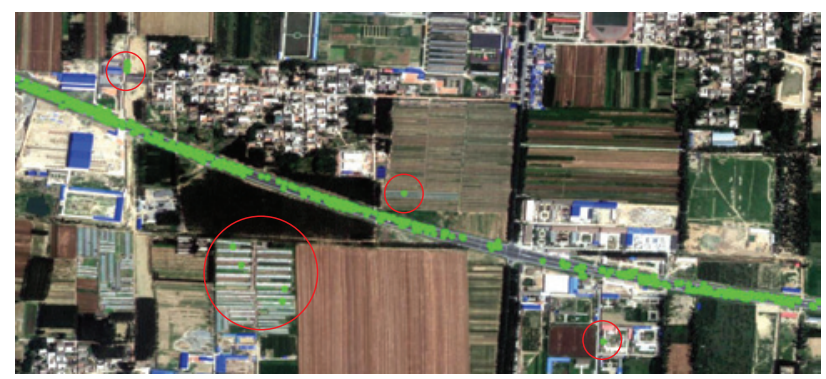

Figure 10. Prediction

Then, use the road texture points to extract the road segments. Build buffer around key points with radius of 8 , about one quarter of the road width. If the segments and the buffer have 50 pixels overlap. The segments are selected and the image is turned into binary image for further operation. The result is shown in 
Figure 11

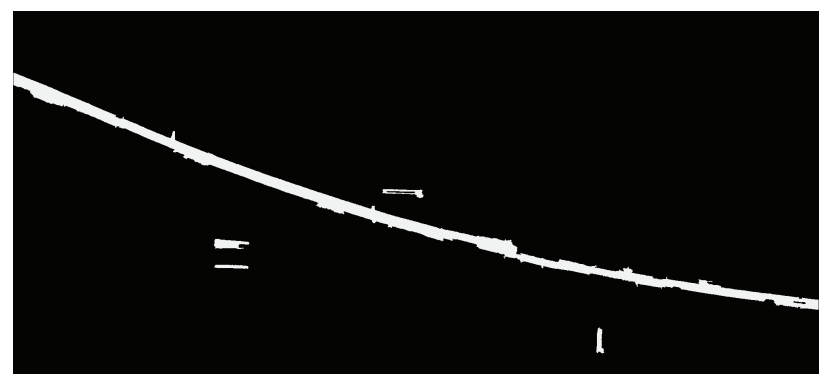

Figure 11. Result of road segments extraction

\section{SHAPE ANALYSIS AND MORPHOLOGY OPERATION}

\subsection{Introduction}

Road has a special shape, it is a long stripe with width. By using shape analysis, the non-road segments can be erased. In this paper, three norms are used. After shape analysis, morphology can be used to prune road segments and extract roads

\subsection{Shape Analysis}

\section{1)Area $S$}

In high resolution image, roads are connected and with a large area. Small non-road object such as buildings can be erased. The threshold is determined by image resolution.

2)Length width ratio $R$ In high resolution image, roads are stripe objects, so the length width ratio of its minimum area bounding rectangle(MABR)(Figure 12) is large.

$$
R=L_{M A B R} / W_{M A B R}
$$

In $4, L_{M A E R}$ and $W_{M A E R}$ are the length and the width of roads minimum area bounding rectangle.

$$
F=S / S_{M A B R}
$$

$\mathrm{S}$ is the area of road, $S_{M A B R}$ is the area of minimum area bounding rectangle, so $F \leq 1.0$. Fullness rate is used to extract complex road network, where $\mathrm{R}$ of roads MABR is not large but $\mathrm{F}$ is very small. If it satisfies the equation 6 , the area is determined as road. $T_{S}, T_{F}, T_{R}$ are thresholds for $\mathrm{S}, \mathrm{F}$ and $\mathrm{R}$.

$$
S>T_{S} \bigcap\left(F<T_{F} \bigcup R>T_{R}\right)
$$

\subsection{Morphology Operation}

Mathematical morphology operation is a theory analyzing spatial structure. It is based on set theory, integral theory and grid algebra. The biggest feature of it is to use shifting and logical operation which is fast and agile rather than other complex operation. In this paper, the open operation is used to prune the coarse edge, then, close operation is used to seal the holes in roads. Finally, thinning operation is used to extract the center line of road.
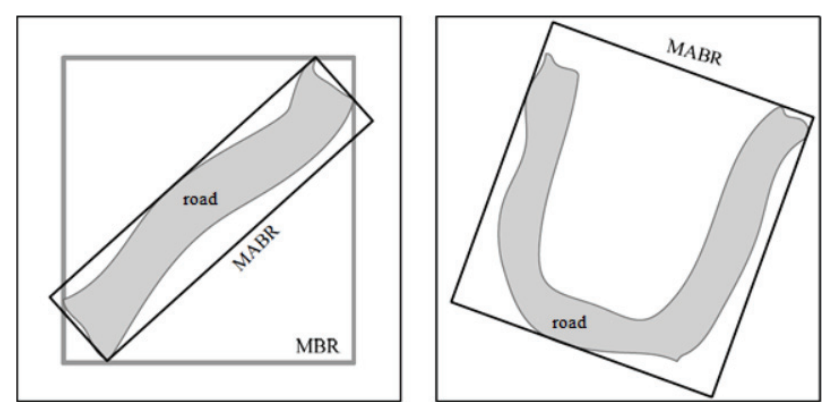

Figure 12. MABR example

\subsection{Experiment and Analysis}

In the part of shape analysis, $T_{S}=1000, T_{F}=0.2, T_{R}=7$. In the Figure 13(b), the MABR of the main road is shown in yellow color. Its $\mathrm{R}$ reaches 17.9 , its fullness reaches 0.21 and the area of road reaches 52023 pixels. Other small erased components parameters are listed below in the order(R,S,F).

$$
(5,939,0.46),(3.75,1456,0.72),(9.89,644,0.92),(3.4,779,0.65) .
$$

In the part of morphology, circle operation element is used. Radius for open operation is 5 which is small in order not to split the road, and radius for close operation is 15 which is bigger in order to fuse some roads that are broken.Figure 13(c)(d) shows the pruned road area and the extracted center line of road. Figure 14 shows the final result overlapped on the original image.

\subsection{Result and Conclusion}

In this paper, we present a work flow of possible road extraction strategy. However, there is still great potential to upgrade each part. ROIs extraction is a good preliminary for further operations. Image segmentations, machine learning techniques together with shape constraints can be effective to choose possible road areas. The morphology operation can be used for segments tuning and centerline detection pretty easily. Overall, this strategy is useful for road extraction applications.

\section{REFERENCES}

Ballard, D. H., 1987. Generalizing the hough transform to detect arbitrary shapes. Pattern Recognition 13(81), pp. 111-122.

Beucher, S., 1994. Watershed, hierarchical segmentation and waterfall algorithm. Computational Imaging \& Vision 2, pp. 69-76.

Bicego, M., Dalfini, S., Vernazza, G. and Murino, V., 2003. Automatic road extraction from aerial images by probabilistic contour tracking. In: Proceedings / ICIP ... International Conference on Image Processing, pp. III-585-8 vol.2.

Chen, Y., Su, W., Li, J. and Sun, Z., 2009. Hierarchical object oriented classification using very high resolution imagery and 1idar data over urban areas. Advances in Space Research 43(7), pp. 1101-1110.

Cheng, J., Guan, Y., Ku, X. and Sun, J., 2011. Semi-automatic road centerline extraction in high-resolution sar images based on circular template matching. In: Electric Information and Control Engineering (ICEICE), 2011 International Conference on, pp. 1688-1691. 


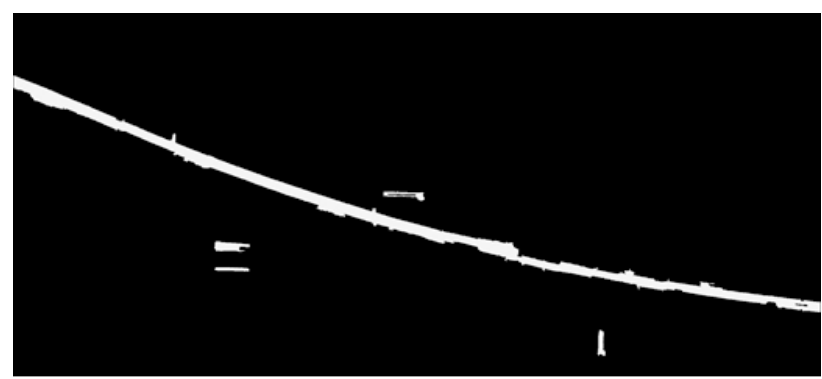

(a)

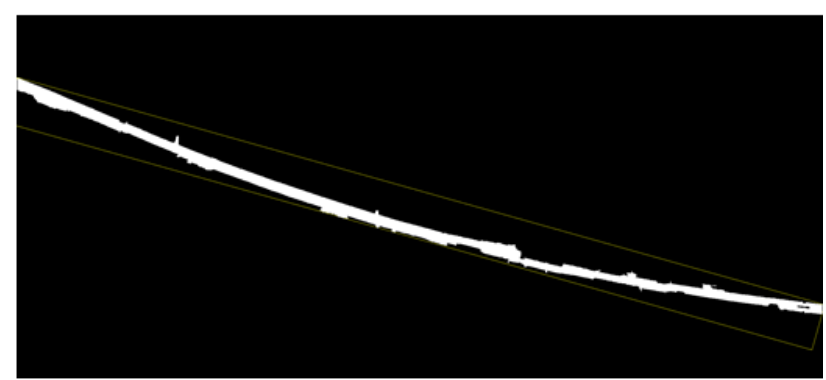

(b)

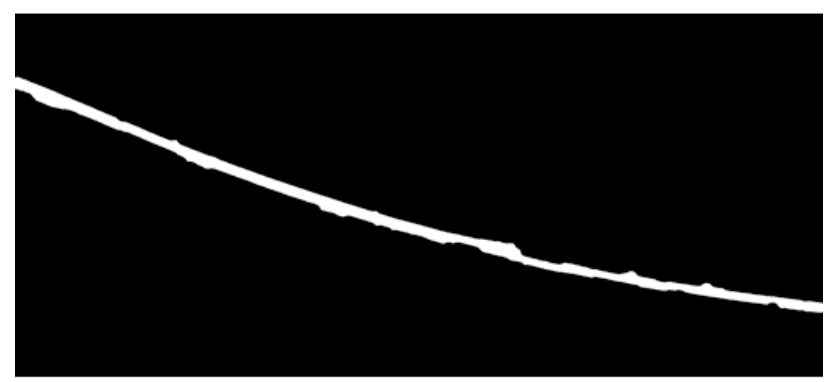

(c)

(d)

Figure 13. (a)Before shape analysis (b)After shape analysis (c)Pruned road (d)Extracted center line of road

Comaniciu, D. and Meer, P., 1997. Robust analysis of feature spaces: color image segmentation. In: Proceedings / CVPR, IEEE Computer Society Conference on Computer Vision and Pattern Recognition. IEEE Computer Society Conference on Computer Vision and Pattern Recognition, pp. 750-755.

D Comaniciu, P. M., 1999. Mean shift analysis and applications. IEEE International Conference on Computer Vision.

Freund, Y., 1995. Boosting a weak learning algorithm by majori-

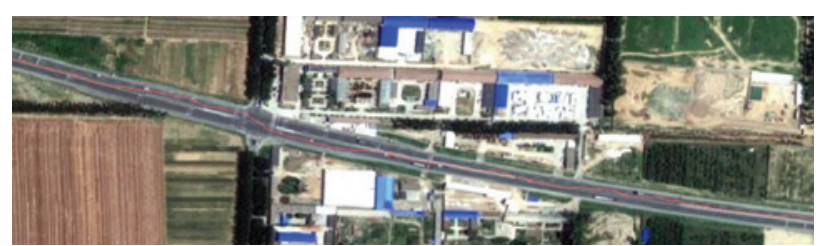

Figure 14. Extracted center line on origin image

ty. IEEE Trans on Acoustics Speech \& Signal Processing 121(2), pp. 256-285.

Fukunaga K, H. L., 1975. The estimation of the gradient of a density function, with applications in pattern recognition. Information Theory IEEE Transactions on 21(1), pp. 32-40.

Gamba, P., Dell' Acqua, F. and Lisini, G., 2006. Improving urban road extraction in high-resolution images exploiting directional filtering, perceptual grouping, and simple topological concepts. Geoscience \& Remote Sensing Letters IEEE 3(3), pp. 387-391.

Hinz, S., Baumgartner, A., Steger, C., Mayer, H., Eckstein, W., Ebner, H. and Radig, B., 2001. Road extraction in rural and urban areas. Ias.informatik.tu.

J, C., 1986. A computational approach to edge detection. Pattern Analysis \& Machine Intelligence IEEE Transactions on pami8(6), pp. 679-698.

Kearns, M., Li, M. and Valiant, L., 1994. Learning boolean formulas. Journal of the Acm 41(6), pp. 1298-1328.

Lindeberg, T., 1994. Scale-space theory in computer vision. Springer Berlin 256, pp. 349-382.

Lowe, D. G., 2004. Distinctive image features from scaleinvariant keypoints. International Journal of Computer Vision 60(2), pp. 91-110.

Meyer, F., 1992. Color image segmentation. In: Image Processing and its Applications, 1992., International Conference on, pp. 303-306.

Singh, P. P. and Garg, R. D., 2013. Automatic road extraction from high resolution satellite image using adaptive global thresholding and morphological operations. Journal of the Indian Society of Remote Sensing 41(3), pp. 631-640.

Sirmacek, B. and Unsalan, C., 2010. Road network extraction using edge detection and spatial voting. In: Pattern Recognition (ICPR), 2010 20th International Conference on, pp. 3113-3116.

Song, M. and Civco, D., 2004. Road extraction using svm and image segmentation. Photogrammetric Engineering \& Remote Sensing 70(12), pp. pgs. 1365-1372.

Sujatha, C. and Selvathi, D., 2015. Connected component-based technique for automatic extraction of road centerline in high resolution satellite images. Eurasip Journal on Image \& Video Processing 2015(1), pp. 1-16.

Valiant, L. G., 1984. A theory of the learnable. commun acm. Communications of the Acm 27(11), pp. 1134-1142.

Vincent, L. and Soille, P., 1991. Watersheds in digital spaces: An efficient algorithm based on immersion simulations. Pattern Analysis \& Machine Intelligence IEEE Transactions on 13(6), pp. 583-598.

Vosselman, G. and Knecht, J. D., 1995. Road tracing by profile matching and kalman filtering. Monte Verit pp. 265-274. 
Wang, Y., Tian, Y., Tai, X. and Shu, L., 2006. Extraction of Main Urban Roads from High Resolution Satellite Images by Machine Learning. Springer Berlin Heidelberg.

Yang, H. and Zu, K. J., 2009. Road extraction from remote sensing imagery based on road tracking and ribbon snake. In: Knowledge Engineering and Software Engineering, 2009. KESE' '09. Pacific-Asia Conference on, pp. 201-204. 